\title{
Regulation of transepithelial transport of iron by hepcidin
}

\author{
NATALIA P MENA, ANDRÉS L ESPARZA and MARCO T NÚÑEZ
}

Biology Department and Cell Dynamics and Biotechnology Research Center, Faculty of Sciences, Universidad de Chile

\begin{abstract}
Hepcidin (Hepc) is a 25 amino acid cationic peptide with broad antibacterial and antifungal actions. A likely role for Hepc in iron metabolism was suggested by the observation that mice having disruption of the gene encoding the transcription factor USF2 failed to produce Hepc mRNA and developed spontaneous visceral iron overload. Lately, Hepc has been considered the "stores regulator," a putative factor that signals the iron content of the body to intestinal cells. In this work, we characterized the effect of Hepc produced by hepatoma cells on iron absorption by intestinal cells. To that end, human Hepc cDNA was cloned and overexpressed in HepG2 cells and conditioned media from Hepc-overexpressing cells was used to study the effects of Hepc on intestinal Caco-2 cells grown in bicameral inserts. The results indicate that Hepc released by HepG2 inhibited apical iron uptake by Caco- 2 cells, probably by inhibiting the expression of the apical transporter DMT1. These results support a model in which Hepc released by the liver negatively regulates the expression of transporter DMT1 in the enterocyte.
\end{abstract}

Key terms: hepcidin, iron absorption, DMT1, Caco-2 cells

\section{INTRODUCTION}

Hepcidin (Hepc) is a 25 amino acid cationic peptide with broad antibacterial and antifungal actions. It is predominantly expressed in the liver and has been detected in blood and urine (Ganz et al., 2004). The human Hepc gene contains three exons that encode a 94-aminoacid pro-hepcidin with a characteristic furin cleavage site immediately $\mathrm{N}$-terminal to the 25-amino acid mature peptide. In addition to the 25 -amino acid form, the urine also contains minor 20and 22-amino-acid forms truncated at the Nterminus (Kulaksiz et al., 2004). Analysis of the sequence of Hepc revealed that the protein contains eight cysteines. Mass spectroscopy and chemical analysis revealed that all of the cysteines are bridged in the sequence, making Hepc a highly constrained peptide (Park et al., 2001).
A likely role for Hepc in iron metabolism was suggested by the observation that mice having disruption of the gene encoding the transcription factor USF2 failed to produce Hepc mRNA and developed spontaneous visceral iron overload (Nicolas et al., 2001).

Lately, Hepc has been considered the "stores regulator," a putative factor that signals the iron content of the body to intestinal cells. Hepc expression and secretion by the liver correlate directly with hepatic iron levels. Once secreted, Hepc produces inhibition of intestinal iron absorption by the enterocyte. Thus, Hepc mediates a regulatory cycle of iron absorption between the liver and the intestine (reviewed in Andrews, 2004).

In this work, we characterized the effect of Hepc produced by hepatoma cells on iron absorption by intestinal cells. To that 
end, human Hepc cDNA was cloned and overexpressed in HepG2 cells, and conditioned media from Hepcoverexpressing cells was used to study the effects of Hepc on intestinal Caco-2 cells grown in bicameral inserts.

\section{RESULTS AND DISCUSSION}

Production of Hepc by HepG2 cells. A full-length Hepc cDNA was cloned in the pcDNA3 plasmid, and the plasmid was transfected in HepG2 cells. The transfected cells showed an increase of Hepc immunofluorescence. Similarly, Western blot of cell extracts from control and Hepc-transfected cells indicated an increase in a $20 \mathrm{KDa}$ band in transfected cells.

${ }^{55} \mathrm{Fe}$ uptake by Caco-2 cells pretreated with Hepc-conditioned media. We studied the effects of $\mathrm{Hepc}$ on ${ }^{55} \mathrm{Fe}$ absorption by intestinal Caco- 2 cells grown in bicameral inserts. Caco-2 cells were incubated for $24 \mathrm{~h}$ with control media or media obtained from $72 \mathrm{~h}$-cultures of Hepcoverproducing HepG2 cells. ${ }^{55} \mathrm{Fe}$ uptake from the apical media was performed (Fig. 1). We found that Hepc decreased apical iron uptake, whereas the transfer step to the basolateral media was not affected. The inhibitory effect on apical ${ }^{55} \mathrm{Fe}$ uptake for Hepc was obliterated when the media was first treated with Sepharose-bound antiHepc antibody.

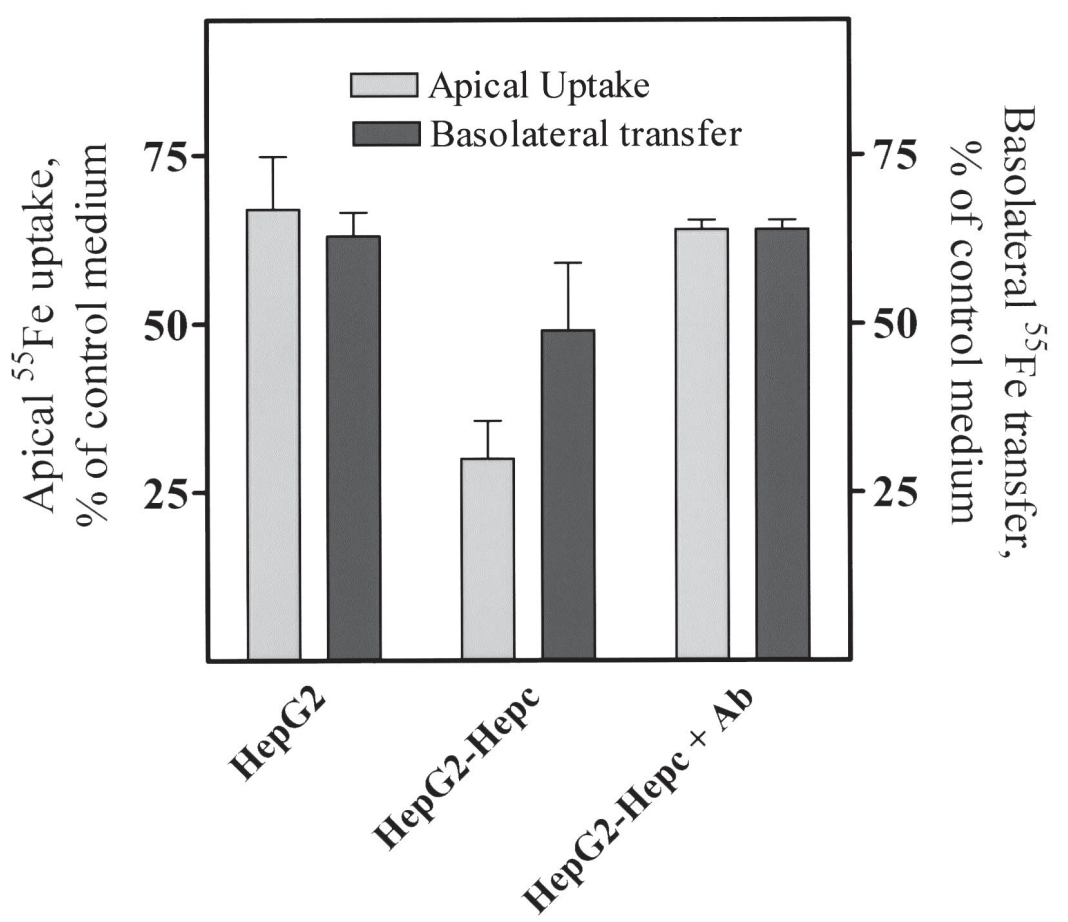

Figure 1. Effect of Hepc in apical ${ }^{55} \mathrm{Fe}$ uptake $(A)$ and basolateral ${ }^{55} \mathrm{Fe}$ transfer $(B)$ by Caco-2 cells. Insert-grown Caco- 2 cells were treated for 24 hours with different culture media in the basolateral chamber. The apical chamber contained standard culture medium. Cells were then exposed for 1 hour to $5 \mu \mathrm{M}{ }^{55} \mathrm{Fe}-\mathrm{NTA}$ (1: 2.2, mol: mol) added to the apical medium, after which cell-associated and basolateral ${ }^{55} \mathrm{Fe}$ was determined. HepG2: medium from untransfected HepG2 cells; HepG2-Hepc: conditioned medium from HepG2 cells transfected with pCDNA3-Hepc; HepG2-Hepc $+A b$ : medium from HepG2 cells transfected with pCDNA3-Hepc that was depleted of Hepc by treatment with anti-Hepc antibody bound to Sepharose. Results were referred to apical ${ }^{55} \mathrm{Fe}$ uptake and basolateral ${ }^{55} \mathrm{Fe}$ transfer by cells cultured in standard culture medium without prior treatment. The results show that Hepc-conditioned medium decreased the apical uptake of ${ }^{55} \mathrm{Fe}$ in Caco- 2 cells. 
Determination of Hepc effect on DMT1 and Ireg1 expression in Caco-2 cells. RNA from Caco- 2 cells treated for 48 hours with Hepc-conditioned medium was analyzed for DMT1 and Ireg1 mRNA expression by semi-quantitative RT-PCR. A two- to three-fold decrease in DMT1 mRNA expression was observed in Hepctreated cells when compared to cells treated with control media. In contrast, no changes in Ireg1 mRNA expression were readily apparent (Mena et al., manuscript in preparation). These results agree with the ${ }^{55} \mathrm{Fe}$ transport experiments, where only apical iron uptake was affected.

In summary, the results indicate that Hepc released by HepG2 inhibited apical iron uptake by Caco-2 cells, probably by inhibiting the expression of the apical transporter DMT1.

These results support a model in which Hepc released by the liver negatively regulates the expression of transporter DMT1 in the enterocyte.
ACKNOWLEDGEMENTS

This work was financed by FONDECYT grant 1040448 and ICM grant P99-031.

\section{REFERENCES}

ANDREWS NC (2004) Iron homeostasis and inherited iron overload disorder: An overview. Clin Invest 113: 12511253

GANZ T (2004) Hepcidin in iron metabolism. Curr Opin Hematol 11: 251-254

KULAKSIZ H, GEHRKE SG, JANETZKO A, ROST D, BRUCKNER T, KALLINOWSKI B, STREMMEL W (2004) Pro-hepcidin: Expression and cell specific localisation in the liver and its regulation in hereditary haemochromatosis, chronic renal insufficiency, and renal anaemia. Gut 53: 735-743

NICOLAS G, BENNOUN M, DEVAUX I, BEAUMONT C, GRANDCHAMP B, KAHN A, VAULONT S (2001) Lack of hepcidin gene expression and severe tissue iron overload in upstream stimulatory factor 2 (USF2) knockout mice. Proc Natl Acad Sci USA 98: 87808785

PARK CH, VALORE EV, WARING AJ, GANZ T (2001) Hepcidin, a urinary antimicrobial peptide synthesized in the liver. J Biol Chem 276: 7806-7810 